Mahmoud FOUAD

\title{
TRANSKULTURELLE ASPEKTE DER SPRACHE ALS IDENTITÄTSSTIFTER IN EMINE ÖZDAMARS "MUTTERZUNGE"
}

Emine Sevgi Özdamar wird 1946 in der Türkei geboren, emigriert 1971 nach Deutschland und ist dort als Schauspielerin, Regisseurin und Schriftstellerin tätig. Ihr Durchbruch in der deutschen Literaturszene erfolgt mit der Verleihung des Ingeborg Bachmann-Preises 1991. Damit wird zum ersten Mal eine fremdsprachliche Schriftstellerin, die auf Deutsch schreibt, mit dem renommierten Preis ausgezeichnet. Özdamars Werk befasst sich vornehmlich mit "Auseinandersetzungen mit der realen Gegenwart, Thematisierung der 'Fremde' und der Fremdheitserfahrung, Öffnung für die multikulturelle Wirklichkeit" und sucht "in der Fremde ihre Heimat in der Fremdsprache Deutsch" (Al-Slaiman 1997: 89). Diese Thematik, die nach der eigenen Heimat und Sprache in der Fremde fahndet, durchzieht Özdamars gesamte Arbeit. "Die Autorin denkt türkisch und schreibt deutsch, und gerade diese Dialektik der (verbalen) Selbstvergewisserung ist es, die das Glück ihrer Prosa ausmacht." (Zimmermann 1986: 7)

In ihrem epischen Band "Mutterzunge" erzählt Özdamar von einer namentlich nicht genannten Protagonistin, die über sich, ihre Gedanken, Liebe und Begierden in der Ich-Erzählform berichtet. Die Ich-Erzählerin verliert ihre Muttersprache in Deutschland. Auf der Suche nach ihrer Identität versucht sie die Wurzeln ihrer Muttersprache aufzuspüren, indem sie Arabisch als "Großvaterzunge" lernt, weil das Türkische früher mit der arabischen Schrift geschrieben wurde. Gerade in den Anfangssätzen wird die Thematik der Erzählung plausibel dargestellt, nämlich die logische Bereitschaft des Menschen zur sprachlichen Transkulturalität. "In meiner Sprache heißt Zunge: Sprache. Zunge hat keine Knochen, wohin man sie dreht, dreht sie sich dorthin." (9) ${ }^{1}$ Man kann Sprachen lernen, doch die erlernte Sprache darf die

1 Die Studie verlässt sich auf Özdamars Prosawerk "Mutterzunge" (Özdamar 2010) als primärliterarisches Korpus. Die nach den diversen Zitaten in Klammern stehenden Zahlen weisen auf die Seitenzahl hin. 
Muttersprache nicht ersetzen, da diese als ein essentieller Bestandteil der menschlichen Identität zu betrachten ist. Denn die Sprache "gibt die Identität von Menschen wieder, ihre Traditionen und ihre Lebenswelt. Wenn Sprache verloren geht, geht auch unwiderruflich Kultur zu Grunde" (Jeppesen 2008). Nicht die Assimilation in der Zielkultur oder der Erwerb der Fremdsprache ist die Problematik, der die türkische Protagonistin begegnet, sondern der Muttersprachenverlust. "Wenn ich nur wüsste, wann ich meine Mutterzunge verloren habe." (9) Der Sprachverlust bezieht sich hier allerdings nicht auf diese Problematik der "sprachlichen Heimatlosigkeit" (Schiskoff 1952/53: 65ff.), die besonders bei den bi- bzw. multilingualen Migranten zweiter Generation auftaucht, da sie in ihrem Alltag in der Fremde nur selten die Möglichkeit haben, ihre Muttersprache zu verwenden.

Die Studie möchte transkulturelle Aspekte der Sprache in Özdamars "Mutterzunge", die als "ein Stück `neue Weltliteratur` auf Deutsch" (Böhm 1999) bezeichnet wird, herausfinden und stellt die erkenntnistheoretische Frage, ob dem Solipsismus alle Sprachtypen, die Mutter- wie die Fremdsprache und zuletzt der Sonderfall der Großvatersprache zugrundliegen und ob diese transkulturellen Aspekte das eigene Identitätsbild schärfen. Dabei wird auf die Identitätsstörung der Protagonistin, die Wesenszüge der Kulturen und den Muttersprachenverlust eingegangen. Um die Ursachen für letzteren zu ermitteln, werde ich die Sprachphilosophie Wittgensteins heranziehen.

\section{1. Überlegungen zur interkulturellen Literatur}

Die Interkulturalität ist kein neues Phänomen. Im Zeitalter der Globalisierung, der universellen Mobilität und der souveränen Kommunikationsmöglichkeiten gewinnt doch die inner- und interdisziplinäre Forschung dieses Phänomens neue Relevanz.

Das literarische Schreiben in einem fremden Land oder in einer fremden Sprache ist kein aktuelles Phänomen der Globalisierung. Viele Autoren fremder Herkunft eroberten bereits die deutsche Literaturszene, wie: Rainer Maria Rilke, Franz Kafka und Franz Werfel aus Tschechien; Paul Celan und Herta Müller aus Rumänien; Adelbert von Chamisso aus Frankreich und Elias Canetti aus Bulgarien. Der Unterschied zwischen diesen Immigranten in die deutsche Literatur und den Anhängern der 
Transkulturelle Aspekte der Sprache als Identitätsstifter in Emine Özdamars „,Mutterzunge“

Migrationsliteratur besteht darin, dass sie vereinzelt in Deutschland eingewandert sind und dass sie sich bald den elitären Intellektuellenkreisen angeschlossen haben, während die neue Welle der Zuwanderer in die deutsche Literatur mit der Gastarbeiterschaft in Verbindung gebracht werden kann; daher auch der Begriff "Gastarbeiterliteratur". "Eine Gesellschaft, die unter Gastarbeiter hartnäckig einen Gast versteht, der arbeiten muss - eine solche Gesellschaft tut sich schwer, die Kultur ihrer Minderheiten zur Kenntnis zu nehmen, die neuen Zungen zu hören." (Böhm 1999) Aber Namen wie Rafik Schami aus Syrien, Rafael Seligmann aus Israel, Aras Ören, Güney Dal, Zafer Senocak oder Dilek Zaptçioglu aus der Türkei gehören zu den meist gelesenen Autoren in der deutschen Literaturszene.

In Verbindung mit Kultur betont das Suffix "inter" das Dazwischen und setzt damit eine Grenze zwischen den Kulturen voraus, die zu überschreiten ist. Diese Auffassung von Interkulturalität als Zwischenräume teilen Blioumi (2002), Rieger/Schahadat/Weinberg (1999) und Görling (1997). Görling erweitert aber diese Einstellung und legt die Interkulturalität als den Begriff aus, "der nicht nur nach den Zwischenräumen zwischen den möglichen kulturellen Formationen fragt und diese hinterfragt, sondern auch die innerkulturellen Einheiten als diskursive Ereignisse begreift, also interkulturell auch nach innen neue Einheiten sucht" (Görling 1997: 52f). Die bipolare Einteilung vom Eigenen und Fremden gewinnt hier eine neue Reichweite, nämlich die gegenseitige Wechselbeziehung eines Diskurses.

Das interkulturelle Positivum der Migration versteht sich als angestrebte Leistung in der Literatur. "Eine avancierte Migrationsliteratur, die Migration nicht als Verlust und Leid, sondern als Stärke versteht und diese Stärke aus dem Oszillieren zwischen dem "Fremden" und dem "Eigenen" schöpft, bietet neue Wahrnehmungsmuster im Hinblick auf eine interkulturelle Wirklichkeit." (Blioumi 2002: 28) In "Mutterzunge" oszilliert die Protagonistin einerseits zwischen dem Eignen und Eignen und andererseits zwischen dem Eignen und Fremden. Erstaunlicherweise vollziehen sich der Verlust und das Leid auf diesen beiden Ebenen. Die Mutterzunge geht in der fremden Kultur verloren und die Großvaterzunge in der eigenen. Die von Blioumi gemeinte Stärke ergibt sich erst am Ende der Erzählung, wenn die Ich-Erzählerin ihre Vergangenheit übergibt und nicht mehr auf ihre Identität durch die arabisch-türkische Brücke eingeht, sondern auf den gegenwärtigen 
Identitätskonflikt durch die deutsch-türkische Brücke. Ein neues Wahrnehmungsmuster ebnet den Weg zu einer interkulturellen Wirklichkeit. Sie sucht nicht mehr nach äquivalenten Entsprechungen der türkischen Wörter im Arabischen, sondern jetzt im Deutschen und mit Deutschen. "'Ruh heißt Seele', sagte ich zu dem Mädchen. 'Seele heißt Ruh', sagt sie." (51) Ein neuer Diskurs entsteht.

\section{2. Überlegungen zur Muttersprache}

Um die Sprachenvielfalt und damit auch die Reichhaltigkeit der Kulturen zu erhalten, haben die Vereinten Nationen das Jahr 2008 zum Internationalen Jahr der Sprachen erklärt. Die Muttersprache als Spiegel von Kultur und Tradition steht im Zeitalter der wirtschaftlichen Krisen vor der Gefahr des Verlustes. In einem Kommentar über den UN-Report zum Jahr der Sprachen wird folgende Gegenüberstellung gemacht: "Ein guter Job oder die eigene Sprache." (Jeppesen 2008) Auf der Suche nach besseren Lebens- und Arbeitschancen verlieren manche Menschen ihre Muttersprache in der Fremde. Im Folgenden wird auf die Muttersprache definitorisch eingegangen.

Die Muttersprache ist als die erste Zugehörigkeit zu einer sozialen Gemeinschaft zu bezeichnen. Sie gilt als "Naturgesetzlichkeit der Zugehörigkeit zu dieser Nation" (Ahlzweig 1994: 143). Die Bezeichnungen "Muttersprache" und "Vaterland" bzw. "Mutterland" unterstreichen die Wechselbeziehung von Heimat und Muttersprache. Auch die Bezeichnung "Deutschland" wie "England", "Frankreich" etc. bekräftigen, dass die Sprachen die Identität für Völker und Länder stiftet, die je nach der Bezeichnung deren Sprache benannt sind. Das Ethnische und das Muttersprachliche gehören zusammen. In "Mutterzunge" sei die Muttersprache für die Migranten "die Milch, die sie aus ihren Müttern getrunken haben" und die wegen der Fremde "aus ihrer Nase rausgeholt" wird (14). Die Ich-Erzählerin lernt Arabisch, um "den Weg zu meiner Mutter und Mutterzunge" (14) zu finden. Damit setzt sie die Muttersprache mit der Mutter gleich. Özdamar intensiviert diese Verbindung mit dem neuen Terminus "Großvaterzunge" (16). Vater, Mutter und Großvater sind Begriffe der biologischen aber auch sprachlichen und nationalen Identität wie Vaterland, Muttersprache und ethnische Vorfahren. 
Transkulturelle Aspekte der Sprache als Identitätsstifter in Emine Özdamars „,Mutterzunge“

Weisgerbers Überlegungen (1938-1957) von der Muttersprache als kulturelle Grundeinstellung werden in zwei Punkten resümiert: "(1) Muttersprache hat eine ,eigentümliche Existenz und Wirklichkeit', nämlich die des Kulturgutes. (2) Muttersprache tritt dem Einzelnen als Norm gegenüber, ,die ihm keine Wahl lässt'; so wird sie ,unabhängig von der Willkür und Zufälligkeit des einzelnen Menschenlebens'." (Ivo 1994: 162) Hier tritt der Sozialisationscharakter der Muttersprache in den Vordergrund. Sie ist eine willkürliche, gesellschaftliche Voraussetzung, damit der Mensch aufgenommen wird.

1973 haben sich deutschsprachige Zeitungen und Rundfunkstationen außerhalb des deutschen Sprachraums zusammengeschlossen und die Internationale Assoziation Deutschsprachiger Medien (IADM) gegründet. Um das Verhältnis zwischen (Mutter)Sprache und Zugehörigkeitsgefühl, Heimat, Integration, Mundarten zu erforschen, wurde 1979 ein Wettbewerb veranstaltet (alle Beiträge in: Nasarski 1981). Für seinen Essay über die (Mutter)Sprache wird Ben-Chorin mit dem ersten Platz prämiert. Mit dem folgenden Satz leitet Ben-Chorin seinen Aufsatz ein: "Aus einem Land kann man auswandern, aus der Muttersprache nicht." (Ben-Chorin 1981: 12) Damit bekräftigt er, dass die Sprache im Vergleich zur Heimat kein geographisch bedingtes Element für die Zugehörigkeit zu einer bestimmten, ethnischen Kultur aufweist. Zwar gehört die sprachliche Herkunft wie die geographische zur Identität des Menschen, doch man kann auf seine geographische Herkunft willig oder widerwillig verzichten, aber niemals auf seine sprachliche Herkunft bzw. Muttersprache. "Ganz heimisch ist man ja doch wohl nur in seiner Muttersprache, zumal wenn sie für uns das Medium schriftlicher Mitteilung geblieben ist. Das blieb das Deutsche für mich." (Ben-Chorin 1967: 48) Das Schrifttum gilt Ben-Chorin als die Voraussetzung, dass man sich in der Muttersprache zu Hause fühlt. Er benutzt den Terminus "anatürliche Muttersprache" (Ben-Chorin 1988: 47) für die Sprache, in der man schreibt, sich wohl und zu Hause fühlt, aber nicht herangewachsen ist.

Den Begriff "Muttersprache" verbindet man aufgrund ihrer Funktion als Sozialisation, ethnische wie nationale Zugehörigkeit, aber auch als schicksalhafte Komponente im menschlichen Leben. Doch man kann sich in der "anatürlichen Sprache" heimischer fühlen als in seiner Muttersprache, was im Folgenden am Beispiel der Protagonistin in "Mutterzunge" eruiert wird. 


\section{Muttersprachliche und fremdsprachliche Sprachidentität}

Die traditionelle Identitätstheorie von Erikson geht davon aus, dass das menschliche Leben ein vollständiger Zyklus ist und sich Identität in jedem dessen Stadien entwickelt, bis eine einheitliche, definitive Identität in einer bestimmten gesellschaftlichen und kulturellen Wirklichkeit vervollständigt wird (Vgl. Erikson 1992: 230). So gilt Einheitlichkeit in der geistesgeschichtlichen Periode der Identitätsforschung als Schlüsselbegriff. Darauf folgt der Begriff der "Pluralität" in der postmodernen Forschung. Dazu ist Keupps Ansatz der Identitätskonstruktion "Das Patchwork der Identität" erwähnenswert (Keupp 1998 u. 1999). Demnach ergibt sich aus der komplexen Vielfalt der modernen, gesellschaftlichen Modelle sowohl interals auch intraindividuelle Pluralität von Identitäten. Multiples Selbst bildet sich in unterschiedlichen, kulturellen, alltäglichen, sozialen Kontexten heraus. Die Summe der situationsbedingten "Teilselbsten" zeigt die menschliche Identität, die sich in stetigem Fortgang gestaltet.

Der Gedanke der Fragmentierung der Identität macht den Weg für die Entwicklung der menschlichen Fähigkeit zur interkulturellen Integration frei. Doch dieser Schritt erfordert das Beherrschen der fremden Sprache als eine essentielle Voraussetzung. Die Marginalisierung dieses konstitutiven Aspekts verhindert die Entwicklung einer notwendigen Teilidentität in der Fremde. Von Natur aus verfügt der gesunde Mensch über Sprechfähigkeit, die mit dem sprachlichen Erwerb multiple Sprachidentität und Mehrsprachigkeit zulässt. Auch innerhalb eines Kulturraums gibt es eine Multiplizität der sprachlichen Identität. Daher variiert sich das sprachliche Verhalten des Individuums in unterschiedlichen, sozialen, intra- sowie interlingualen Situationen. Aber eine wesentliche Voraussetzung für die Mehrsprachigkeit liegt am individuellen Willen und Bedarf, eine Sprache oder sprachliche Varietät zu erwerben.

Die Muttersprache aber unterscheidet sich von dieser Auffassung. Der Mensch benötigt die Muttersprache für seine "primäre Sozialisation", die "sowohl die Grundlagen für die Entwicklung einer stabilen Persönlichkeit als auch [...] die Eingliederung in die Gesellschaft" bestimmt (Steinmüller 1984: 242). Man kann sie nicht ablehnen, da sie "mit den affektivemotionalen, den kognitiven und den sozialen Aspekten der Persönlichkeitsbildung" 
Transkulturelle Aspekte der Sprache als Identitätsstifter in Emine Özdamars „,Mutterzunge“

(Steinmüller ebd.) eng verbunden ist. Die Muttersprache ist ein Zwang für die menschliche Sozialisation, während der Erwerb anderer, sprachlicher Varietäten darunter der Fremdsprache auf einer Selbstentscheidung beruht. Die Ich-Erzählerin hebt dieses Eigenwillige durch die Akzentsetzung auf das Personalpronomen der ersten Person Singular hervor. "Eine von mir gut gelernte Fremdsprache." (9/11, eigene Hervorhebung).

Noch ein differenzierender Faktor besteht in der Aneignungsphase. Die Muttersprache erwirbt der Mensch in der Kindheit und andere, sprachliche Varietäten später bzw. nicht früher als die Muttersprache. "Meine deutschen Wörter haben keine Kindheit." (Özdamar 2011: 131) Die Muttersprache eignet man sich "als Kind von den Eltern" (Wermke 2006) souverän an. Der Erwerb der Fremdsprache vollzieht sich hingegen nur im konkreten, erfahrungsmäßigen Zusammenkommen mit der Sprache. "Aber meine Erfahrung mit deutschen Wörtern ist ganz körperlich. [...] Die deutschen Wörter - entweder habe ich sie am Theater selbst gespielt oder sie von den Schauspielerfreunden gehört." (Özdamar 2011: 131f) Diese physische Natur der Fremdsprache realisiert sich bei der Ich-Erzählerin noch im Erwerb der arabischen Sprache, deren Buchstaben sie auch ganz körperlich empfindet. "Es kamen aus meinem Mund die Buchstaben raus. Manche sahen aus wie ein Vogel, manche wie ein Herz, [...] wie eine Karawane, manche wie schlafende Kamele, manche wie ein Fluss, [...] wie laufende Schlangen." (19) Diese Bilder arabischer Buchstaben beziehen sich nicht nur auf den Bereich des Grapheologischen bzw. darauf, wie die Buchstaben graphisch verschriftlicht werden, sondern auch auf den Bereich des Akustischen, d.h., wenn sie die Buchstaben ausspricht, verkörpern sie sich für die Protagonistin in solchen Bildern. Die Bilder arabischer Buchstaben werden komplexer: Manche sehen für sie aus wie "unter den Regen und Wind frierende Granatapfelbäume [...] manche wie in einem türkischen Bad auf einem heißen Stein sitzender dicker Frauenarsch [...] Ich ging mit Kamelen und weinenden Frauenaugen wieder. [...] Über Tüchern warten die Buchstaben auf mich" (19f).

Die sprachliche Identität als Teilidentität gliedert sich in sprachliche Subidentitäten aufgrund der Sprachvarietät ein. Die muttersprachliche Teilidentität, die in der Kindheit beigebracht wird, ist ein Muss, damit der Mensch sozialisiert wird. Die fremdsprachliche basiert hingegen auf der 
Eigenwilligkeit und bildet sich in konkreten Gegebenheiten. Daher wird beim Erwerb der Fremdsprache ihre physische Natur in den Vordergrund gerückt.

\section{Fragmentarische Sprachidentität}

Normalerweise soll das muttersprachliche Teilselbst als die am meisten vollständige Sprachidentität betrachtet werden, aber wenn dieses solide Teilselbst gestört oder sogar verloren wird, steht es gleich neben anderen Sprachidentitäten.

Die Suche nach der verlorenen Muttersprache begleitet die ganze Handlung in "Mutterzunge" mit der Hoffnung der Protagonistin, diesen Moment des Verlustes eruieren zu können. "Vielleicht habe ich meine Muttersprache im IC-Restaurant verloren. [...] Vielleicht habe ich dort - am Kölner Dom - meine Mutterzunge verloren." (13) Sie sucht nicht mehr ihre ganze Muttersprache, sondern bloß einzelne Wörter, die besondere Erinnerungen an die mit der Muttersprache verbundene Vergangenheit hervorrufen, wie Görmek (sehen), Kaza geciremek (Lebensunfälle erleben) und ISCI (Arbeiter) (12). Die Muttersprache tritt stellenweise fragmentarisch auf. Auch Arabisch, ihre Großvaterzunge, bedeutet für sie einzig das alte Schriftbild ihrer Muttersprache, darin fahndet sie ebenfalls nach einzelnen Wörtern. Bis zum Schluss bleibt sie im Bereich des Fragmentarischen ohne klare Vision, was die Muttersprache betrifft. Zum Schluss heißt die Antwort auf die Frage "Was machen Sie in Deutschland?" [...] "Ich bin Wörtersammlerin" (50f). Ganze Sätze sagt sie nur auf Deutsch. Die verlorene, türkische Sprache bedeutet für die Identitätssituierung der Protagonistin einen psychischen Zwischenraum von Vergangenheit, vertreten durch die arabische Sprache und die Familie, und Gegenwart, präsentiert durch die Fremde und die deutsche Sprache.

Den Versuch, Arabisch zu lernen, rechtfertigt sie mit dem beinahe unwahrscheinlichen Grund: "Wenn mein Großvater und ich stumm wären und uns nur mit Schrift was erzählen könnten, könnten wir uns keine Geschichten erzählen." (14) Dann erwähnt sie, was sie zum Erlernen der arabischen Sprache am meisten veranlasst hat, nämlich die Suche nach der gestörten, muttersprachlichen Identität."Vielleicht erst zu Großvater zurück, dann kann ich den Weg zu meiner Mutter und Mutterzunge finden." (14) Mit dem Verbot 
Transkulturelle Aspekte der Sprache als Identitätsstifter in Emine Özdamars „,Mutterzunge“

der arabischen Schrift verliert ihre muttersprachliche Identität einen ihrer Bestandteile und bleibt bruchstuickhaft. "Dieses Verbot - der arabischen Schrift - ist so, wie wenn die Hälfte von meinem Kopf abgeschnitten ist." (31)

Obwohl sich Türken und Araber in Vielem ähneln, es werden etwa zehn sprachliche Übereinstimmungen (43f) gezählt, unterscheiden sich die Vertreter beider Sprachen doch am Ende. Eine neue Realität entsteht, wo sich das Fremde "vereinheimischt" und das Einheimische verfremdet wird. Die Stelle der Muttersprache erobert eine neue Sprache, die die Ich-Erzählerin mit ihrer eignen Muttersprache verwechselt. "'Bruder, zieh es an`. Mahirs Bruder sah mich an, als ob ich eine fremde Sprache spreche." (14) Die Protagonistin geht davon aus, dass sich Türken in Deutschland miteinander selbstverständlich auf Deutsch verständigen, was für sie zweifelsohne eine Fremdsprache bedeutet. Der Verlust der Muttersprache bewirkt aber, dass das Deutsche keine Fremdsprache mehr ist.

\section{Wesenszüge der Kulturen}

"Mutterzunge" ist ein Panorama, durch das Bilder von der deutschen, arabischen und türkischen Kultur dargestellt werden. Der Text weist auf kulturell phänomenologische Besonderheiten hin.

Gegen eine aus der Heimat stammende, soziale Klassifizierung aufgrund der Sprachidentität, hier dem Dialekt, was selbstgebaute Mauer zwischen Türken errichtet, rebelliert Özdamar. "Meine Mutter stand mir gegenüber, aber ich konnte sie nicht umarmen. Zwischen uns stand eine Mauer aus dem fremden Dialekt, [...] Meine Mutter sagte: ,Sprich nicht so, du musst wieder istanbultürkisch, sauberes Türkisch sprechen. [...] Wenn du so anatolisch sprichst, werden alle zu dir Bauer sagen. ' Ich machte wieder meine Arme auf [...] zwischen uns diese Dialektmauer." (Özdamar 1992: 53) Beeinflusst von dieser sozialen Einstellung wird die Protagonistin durch ein Minderwertigkeitsgefühl belastet. Sie möchte ihre herkömmliche und berufliche Identität leugnen, um Ansehen zu wecken. "Ich überlege mir, ob ich sagen soll: ,Ich bin Italienerin.' Meinen Pass, in dem Beruf ISCI (Arbeiter) steht, will ich verstecken, ich denke, wenn ich mich als Studentin oder als Künstlerin ausweisen kann, komme ich durch die Kontrolle durch." (12) 
Im ganzen Text steht die Bezeichnung "arabische Wörter" oder "arabische Sätze" für Koranverse (27, 34f), auch die Erlebnisse der Araber werden mit Korangeschichten interpretiert und erklärt (39f, 40), das Türkische wird hingegen durch Lieder präsentiert (35). Erstaunlich ist, dass der Lehrer für Arabisch für seine Lernenden nur den Koran als Lernstoff auswählt, als wäre in der arabischen Sprache nichts anderes zu unterrichten als der Koran. Wenn Abni Abdullah bei einem romantischen Gespräch mit der Ich-Erzählerin eine Kassette laufen lässt, gibt diese Koranrezitationen (26) wieder. Aber wenn der türkische Vater der Protagonistin im arabischen Radio nach Sendern sucht, stellt er es auf arabische Lieder ein (30). Der arabisch-israelische Konflikt und dessen Opfer werden auch im Text thematisiert (17, 31). Dabei wird die arabische Neigung zum Frieden hervorgehoben (17).

Araber wie Türken leiden unter der politischen Unterdrückung seitens ihrer Regierungen. Abni Abdullah erzählt: "Wenn ich verletzt war, sprach ich etwas laut gegen die Regierung, sie beschuldigt mich, ich sei von den fanatischen Islambrüdern." (17) Und die türkische Protagonistin erzählt weiter mitleidig: "Ich bin für meine Regierung Kommunistin." (17)

Deutschland tritt als das Land der Demokratie aber auch des unterdrückenden Kapitalismus auf: "Hier in Deutschland aber kann ich in den Park gehen und meine Meinung laut sagen, hier gibt es Demokratie. [...] Und wie viele Mal sind Sie in diesen neuen Jahren in den Park gegangen und haben Ihre Meinung laut gesagt, das Geld hat keine Angst hier, es hat Zähne." (17) In Deutschland hat alles seinen Preis auch die Geschichte: "Meine Freunde tun in eine Filmzeigermaschine Kurzfilme von 1936. Sie hatten sie von einer alten Frau gekauft, ihre Jugend." (21)

Dazu steht Deutsch im Text für Distanz, Formalität, Sinnlichkeit und hohe Arbeitsamoral: Wenn sich der arabische Lehrer dafür entscheidet, keinen Geschlechtsverkehr mehr mit der Protagonistin zu haben, begrüßt er sie auf Deutsch: ",Ich wünsch Dir einen schönen Nachtmittag', sagte er. Ich sagte: ,Das ist sehr deutsch.' Er sagte: ,Trotzdem wünsche ich dir einen schönen Nachmittag." Ging." (42) Vor dieser Entscheidung haben sie sich auf Arabisch begrüßt: "Selamünaleyküm." "Aleykümselam." (16)

Im Moment der sinnlichen Trennung möchte die Ich-Erzählerin das Haus verlassen, darauf reagiert Abni Abdullah mit dem folgenden Urteil: "Ja, wie die Deutschen, nicht, kein Sex, tschüß." (44) Ein Wesenszug für das Leben im 
Transkulturelle Aspekte der Sprache als Identitätsstifter in Emine Özdamars „,Mutterzunge“

Westen spiegelt sich in den sexual freien Beziehungen wider. Abni Abdullah als Vertreter der islamischen Kultur empfindet große Reue und hat Angst vor einer Gottesstrafe, weil er mit der Protagonistin geschlafen hat. "Ich will die heilige Liebe, reine Liebe. Wenn ich mit dir weiterschlafe, mein Körper wird sich ändern." (44f) Während sich der arabische Lehrer an die religiösen Gebote hält, befindet sich die türkische Ich-Erzählerin in einer Zwischensituation. Ein Konflikt zwischen zwei Lebenseinstellungen, einer gegenwärtigen, sinnlichen, für die die deutsche Lebensauffassung steht, und einer traditionellen, seelischen, die die islamische Kultur repräsentiert, bestimmt ihre Persönlichkeit. In ihren Begierden stellt sie sich einen sinnlichen Abni Abdullah vor, der in ihren Körper eindringt (u.a. 22, 33, 34), und wenn Abni Abdullah mit der Sinnlichkeit aufhört, spürt sie in sich einen anderen, seelischen Abni Abdullah (51). In diesem Moment erinnert sie sich an das Wort "Ruh", das Seele heißt. "Und Ibni Abdullah, die Seele in meiner Seele, dachte ich und erinnerte mich noch an ein Wort in meiner Mutterzunge: Ruh." (51, Hervorhebung im Original) Er ruft in ihrem Gedächtnis das Wort "Seele" und deren Erhabenheit hervor, die sich dem Körper und dessen Begierden entgegensetzt. Projizierend auf die Korangeschichte von Joseph und Zeliha erzählt Abni Abdullah von seiner ersten sexuellen Erfahrung, die er in seinem achtundzwanzigsten Lebensjahr hatte und zu der er erzwungen war. "Meine Nachbarin hat mich zum Beten eingeladen. [...] Die hat den Zimmerschlüssel aus ihrem Fenster rausgeschmissen und mich entjungfert, ich lief im Zimmer um her, aber sie hat mich geschnappt." (37) Auf der anderen Seite hat die türkische Protagonistin ihre erste, sexuelle Erfahrung in Deutschland konkret geplant mit der Begründung: "Ich muss heute nacht von dieser Jungfernhaut mich retten." (37) Die orientalische Auffassung von Ehre, mit der sie von der Heimat gekommen ist, soll im offenen Deutschland entlastet werden. Sie plant eine einmalige Liebesnacht mit einem hinkenden Mann, um die erwünschte Rettung von der Tradition zu erlangen.

Die berühmte, deutsche Arbeitsmoral betont der Text ebenfalls. Eine ähnliche Situation wird in Deutschland und der Türkei vergleichend dargestellt, um die Unterschiede zwischen den beiden Kulturen hervorzuheben. "Meine Stiefel knirschen wie von einem Werbefilmcowboy. Die Kantinenarbeiter rauchen, reden über Töpfe und Teller, draußen warten Bierfässer, Gasflaschen, jeder redet über Arbeit." (13) Obwohl die Ich-Erzählerin die Aufmerksamkeit mit 
dem Geräusch ihrer Schuhe lenkt, spricht jeder im Gasthaus über die Arbeit. Auf der anderen Seite betritt sie in der Türkei ein Lokal ganz leise. Trotzdem mischt sich die Arbeiterin sofort in ihr persönliches Leben ein. "Geh auf Fingerspitzen in die Türkei, in einem Diwan sitzen, Großmutter neben mir. In Istanbul im Türkischen Bad sitzen. [...] Mich wusch mal eine Zigeunerin, sie fragte mich: ,In welchem Haus arbeitest du, meine Schöne? '" (13)

\section{Transkulturelle Aspekte der Sprache als Identitätsstifter}

\subsection{Abweichende Grammatikalität in der Fremdsprache}

Özdamars kreativer, eigenständiger Sprachstil vermeidet weder "grammatische, lexikalische oder syntaktische Regelverstöße, noch übergangslose, unerwartete wörtliche Übersetzungen aus dem Türkischen, Einflechtungen von Koransprüchen, Märchen, Sentenzen und Sprichwörtern ihrer Heimat" (Dufresne 2006: 2). Die Autorin beabsichtigt eine Betonung des Fremdsprachlichen, empfindet dabei gewisses Wohlbefinden, dieses Abweichen vom fehlerlosen Beherrschen der Sprache hervorzuheben und damit die eigenwillige, selbstentscheidende Tätigkeit mit der Fremdsprache und das identitätsstiftende Bestreben mitten in der Fremde. "Ein japanisches Sprichwort sagt: nur die Reise ist schön - nicht das Ankommen. Vielleicht liebt man an einer fremden Sprache genau die Reise. Man macht auf der Reise viele Fehler, aber man kämpft mit der Sprache, man dreht die Wörter nach links und rechts, man arbeitet mit ihr, man entdeckt sie." (Özdamar 2001: 131) Grammatische Fehler - wie: "Ibni Abdullah [...] legte seinen Mund zwischen meiner Wange und Mund" (24), "dieser Sätze, von der Mutter eines Aufgehängten, erinnere ich mich auch nur so, als ob sie diese Wörter in Deutsch gesagt hätte" (11) und "Gottseidank ich gehöre noch zu einer Generation, die mit vielen arabischen Wörtern aufgewachsen ist" (31) vergegenwärtigen das Fremdsein der Autorin durch die Sprache und verschaffen ihr gleichzeitig neue Identität als Kämpferin in der Fremdsprache, wie sie in einem Gespräch berichtet: "Die Fehler sind meine Identität. Fünf Millionen Menschen, die hier leben, sprechen mit diesen Fehlern. Das ist eine neue Sprache." (Özdamar 1994: 81) In ihrer Beschreibung eines autobiographischen Erlebnisses betont sie den Gedanken, dass sprachliche Fehler den Migranten ihre Identität in der Fremde beimessen. "Die Fehler, die 
Transkulturelle Aspekte der Sprache als Identitätsstifter in Emine Özdamars „Mutterzunge“ wir in der deutschen Sprache machten, waren wir, wir hatten nicht mehr als unsere Fehler." (Özdamar 1993)

\subsection{Relativierung heiliger Texte}

Auch in den eingeführten, arabischen Überlieferungen fehlen Abweichungen nicht, in Form von falschen Übersetzungen der Koranverse, Verwechselung von Koranversen und -auslegung, Überschneidungen von Koranversen mit Liedern und anderen Koranversen: Die ganze Sure Nummer 82 "Der Riss" wird in den Seiten 20 und 25 übersetzt. Doch in der Übersetzung werden Wörter verwechselt wie Meer und Wasser. "Wenn sich die Wasser vermischen." (20) Die richtige Übersetzung lautet: "Wenn die Meere wütend und alle Sperren sprengen." (Maher 2007: 1271)

Der arabische Lehrer erzählt der Protagonistin die Geschichte von Zeliha und dem Propheten Yusuf (39f). Die Geschichte wird so dargestellt, als wäre sie aus dem Koran zitiert, dessen Verse im ganzen Text in Anführungszeichen gesetzt werden. Diese dargestellte Geschichte ist aber einer überlieferten Auslegung der Sure entnommen (Vgl. Al-Kortoby 2006: 310). Mitten an dieser Stelle treten plötzlich richtige Verse aus dem Koran auf, die gleich markiert sind wie der Auslegungstext. Die Grenze zwischen Koran und Auslegung wird aufgehoben. Dieses Motiv wirkt weiter, wenn die IchErzählerin die Lernenden hinter dem Vorhang hört, diese rezitieren den Vers 19 aus der Sure 33 "Die Verbände" und die Protagonistin mischt die gehörten Phrasen mit anderen der Verse 95 und 96 aus der Sure 16 "Die Bienen", und damit entsteht ein Dialog zwischen den diversen Versen (27). Dieselbe Technik vollzieht sich an anderer Stelle, wo sich die Koranverse 105-107 aus der Sure 11 "Hûd" mit einem türkischen Liebesleid so mischen, dass sich beide Textsorten ergänzen und ineinander kohärent führen. Hier werden Phrasen aus den Versen weggelassen, die im Kontext mit dem Lied nicht in Einklang stehen (34f).

Mit dieser Relativierung des heiligen Textes durch Weglassen, Ergänzen oder Verwechseln entfernt sich Özdamar von der islamischen, arabischen Identität und bekräftigt die Vorstellung einer islamisch liberalisierten, türkischen Identität, die u.a. als Resultat der Säkularisierung 
und der Einführung der neuen, lateinischen, islamfremden Schreibornamentik in der Türkei einzuschätzen ist.

\subsection{Verflechtung multilingualer, transkultureller Momente}

Im Rahmen des Zusammenkommens multilingualer und transkultureller Momente findet ein Diskurs zwischen dem Türkischen und dem Arabischen statt. Dabei mischen sich die Koranverse, die als Repräsentanten für das Arabische stehen, und ein türkisches Lied. Die Muttersprache der IchErzählerin überschneidet sich mit der arabischen Fremdsprache, wobei sich beide Sprachen ergänzen. Während der Leser dieses Zusammenkommen beider Sprachen auf Deutsch wahrnimmt: "Koran: ,Wenn jener Tag kommt, dann wird keine Seele sprechen. ' Türkisches Lied: ,Mein Leben lang will ich deine reine Liebe in meinem Herzen lassen. ' [...]" (34f) Dieser Austausch wiederholt sich neun Mal an dieser Stelle, um die Wechselwirkung beider Sprachen zu betonen, die ihren Anhängern eine gewisse Identität stiftet. Das Arabische gewinnt seine Identität durch den Koran, das Türkische durch Lieder und das Deutsche durch die geschlagene Brücke, die die Anhänger verschiedener Sprachen und Herkunft aufgrund deren Aufenthalts in Deutschland ermöglicht, sich gegenseitig zu verständigen. Dabei erfüllt das Deutsche eine transkulturelle Rolle für die Migranten selbst. Immer wenn dieses gegenseitige Zusammenkommen der Sprachen auftaucht, wird auf ein Hindernis hingewiesen nämlich den Vorhang, der zwischen der Protagonistin und der arabischen Sprache stattfindet. "Ich lernte sehr leise, hinter dem Vorhang waren sie laut, ihre Sätze und meine Sätze mischten sich." (27 eigene Hervorhebung) "Ich lernte nicht nur mein Schriftstück, lernte die Wörter, die hinter dem Vorhang gesprochen wurden, mit, dann kam wieder ein türkisches Lied, und das mischte sich in die arabischen Wörter." (34 eigene Hervorhebung) Der Vorhang kann die beiden Sprachen doch nicht trennen, solange ein aktioneller Wille ihn zu überwinden versucht. Es ist der Wille der Lernenden, die verschiedenen Sprachen angehören und trotzdem Sätze bzw. Lieder in arabischer Sprache artikulieren.

An anderer Stelle übersetzen die beiden Sprecher füreinander ihre muttersprachlichen Wörter nur durch Deutsch. "Wie heißt Esrar? ,Geheimnisse.' ,Bei uns auch. Was heißt Evham?' ,Irrige Vorstellung.' ,Bei 
Transkulturelle Aspekte der Sprache als Identitätsstifter in Emine Özdamars „,Mutterzunge“ uns auch '[...]" (43-Hervorhebung im Original) Wieder taucht das Deutsche als transkulturelle Brücke zwischen den beiden Fremdsprachlern auf. Am Ende der Erzählung begegnen sich die türkische und die deutsche Sprache. Auf die Frage eines deutschen Mädchens "Was machen Sie in Deutschland?" antwortet die Ich-Erzählerin: "Ich bin Wörtersammlerin", dann sagt sie zu ihm: "Ruh heißt Seele", und das Mädchen erwidert: "Seele heißt Ruh." (51 Hervorhebung im Original) Während die türkische Sprecherin die Entsprechung eines türkischen Wortes im Deutschen findet, zeigt die deutsche Sprecherin ihr Interesse, geht von ihrer Muttersprache aus und zielt auf die sprachliche Entsprechung im Türkischen. Diese korrelative Übersetzung, türkisch-deutsch und deutsch-türkisch, zeigt einen transkulturellen Aspekt der Sprache und deren Sprecher, die ein gegenseitiges Interesse zeigen.

Ein anderer, transkultureller Aspekt des Textes spiegelt sich in der Mischung der arabischen, türkischen und deutschen Wörter wider. Der Makrotext der Erzählung ist auf Deutsch, doch treten darin türkische Wörter und deren sprachliche Äquivalente im Arabischen auf der Mikroebene auf. Deutsch ist die überbrückende Sprache, durch die andere Sprachen der Kommunizierenden übersetzt werden. Deutsch ist die einzige komplette Tatsache mitten zwischen Fragmentarischem, dem Türkischen als Erinnerungsmomenten ohne Gegenwart und dem Arabischen - in dessen Verhältnis zum Türkischen - als grafischer Schrift ohne Laut. Ein trilinguales, transkulturelles Schreiben auf Deutsch geht im Text vonstatten.

\section{7. $\quad$ Sprachkonstituierung und Sprachverlust nach Wittgenstein}

\subsection{Sprache und Denken}

In seinem "Tractatus logica-philosophicus" setzt Wittgenstein den Akzent auf die Sprache als das Präsentationsmedium für menschliches Denken. "Die Logik erfüllt die Welt; die Grenzen der Welt sind auch ihre Grenzen. [...] Was wir nicht denken können, das können wir nicht denken; wir können also auch nicht s a g e n, was wir nicht denken können." (Wittgenstein 1922: 5.61, Hervorhebung im Original) ${ }^{2}$ Wittgenstein betrachtet die Welt als

2 Nach den zitierten Tractatus folgt deren Nummerierung nach Wittgenstein, die im Gegensatz zur Seitenzahl in allen Ausgaben einheitlich ist. 
die rationale, alles, was außerhalb der rationalen Betätigung steht, gehört nicht zur Welt und kann in Folge dessen nicht verbalisiert werden, da die Sprache das Mentale in der Welt präsentiert und deren Grenze die Grenzen der Welt bedeutet. Er unterscheidet allerdings zwischen der mentalen und der sprachlichen Ebene. Die sprachliche Ebene gilt als Konkretisierung der geistigen Tätigkeit. Genau das betont auch Özdamar in "Mutterzunge": "Was der Kopf denkt, sagt der Mund." (110) Das Sprechen als konkreter Akt setzt dementsprechend das Denken als abstrakten Akt voraus. "Im Satz drückt sich der Gedanke sinnlich wahrnehmbar aus." (Wittgenstein 1922: 3.1) "Der Satz konstruiert eine Welt mit Hilfe eines logischen Gerüstes [...]" (Wittgenstein ebd.: 4.023) Dazu sind die muttersprachlichen Wörter nicht mehr fähig. Denn "in der logischen Syntax darf nie die Bedeutung eines Zeichens eine Rolle spielen" (Wittgenstein ebd.: 3.33). Die beiden Sätze auf Türkisch im ganzen Text stammen vom Großvater. Sie sind Aphorismen, die in sich eine bestimmte Logik beinhalten. Erkenntnistheoretisch ist die Verbindung zwischen Sprache und Denken eine synthetische, wahrnehmungsmäßige Erfahrung. Die Subjektivität dieses Urteils über Sprache und Denken wird im folgenden Abschnitt ausgeführt.

\subsection{Subjektivität des Urteils über Sprache}

"Die Grenzen meiner Sprache bedeuten die Grenzen meiner Welt." (Wittgenstein ebd.: 5.6) Die Welt der Protagonistin ist deutsch geworden, ihr Denken ist auf Deutsch eingestellt, und das geschieht auf Kosten des Türkischen, was der Mutter der Protagonistin gleich am Anfang der Erzählung auffällt. "Weißt du, du sprichst so, du denkst, dass du alles erzählst, aber plötzlich springst du über nichtgesagte Wörter, dann erzählst du wieder ruhig. [...] Du hast die Hälfte deiner Haare in Alamania gelassen." (9)

Der Aufenthalt in Deutschland hat Konsequenzen mit sich gebracht: Die Erinnerungen an die Vergangenheit beginnen sich auf Deutsch zu manifestieren. Die Ich-Erzählerin erinnert sich an die Sätze einer türkischen Mutter, "als ob sie diese Wörter in Deutsch gesagt hätte" (11). Die Welt der Protagonistin hat sich so eingedeutscht, dass ihr die Muttersprache in Schrift und Laut als eine Fremdsprache erscheint. Türkische Sätze "kamen in meine Ohren wie eine von mir gut gelernte Fremdsprache" (9) und 
Transkulturelle Aspekte der Sprache als Identitätsstifter in Emine Özdamars „,Mutterzunge“

Zeitungsausschnitte "kamen auch in meine Augen wie eine von mir gut gelernte Fremdsprache" (11). Der arabische Lehrer hat sie nach der türkischen Entsprechung für ein arabisches Wort gefragt. Die Antwort kommt aber auf Deutsch, ihre Muttersprache versagt: ",Was heißt in Türkisch Merhamet?" ,Erbarme‘." (44) Özdamar entwirft durch diese sprachlichen Verwechselung "keine neu zu definierende deutsch-türkische Kultur, sondern eine Freiheit, die sich der Aufgabe stellt, neue Verhaltensmuster und Normen in der Gestaltung des Lebens in der interkulturell bestimmten Welt der Gegenwart zu entwickeln" (Hofmann 2006: 225f). Die subjektive Freiheit in der sprachlichen Gestaltung erlaubt die hybride Begegnung dreier Sprachen in "Mutterzunge", deren Schriftstellerin türkisch, deren Sprache Deutsch ist und deren Deckelblatt die arabische Schrift zeigt.

\subsection{Vom Solipsismus zur koordinierten Wirklichkeit und Sprachverlust}

Wittgenstein hebt die Subjektivität dieser Urteile über Sprache, Denken und Welt hervor: "Ich bin meine Welt." (Wittgenstein 1922: 5.63) Die Welt ist ein Begriff, dem durch individualistische Einstellung und Erfahrung seine Bedeutung und Grenze zugewiesen werden. Die Welt ist somit ein mentaler und nicht ein territorialer Begriff. "Was der Solipsismus nämlich meint, ist ganz richtig, nur lässt es sich nicht sagen, sondern es zeigt sich. Dass die Welt $\mathrm{m}$ eine Welt ist, das zeigt sich darin, dass die Grenzen d e r Sprache (der Sprache, die allein ich verstehe) die Grenzen meiner Welt bedeuten." (Wittgenstein ebd.: 5.62, Hervorhebung im Original)

\subsubsection{Erlebte Sprache}

Mit dem Solipsismus ${ }^{3}$ schafft man sich eine eigene, gültige Wirklichkeit mit deren Bewusstseinsinhalten. Dieses "Eigenpsychische" wird als "eine methodische Beschränkung auf das tatsächliche Erlebte"

Der Solipsismus ist die philosophische Position, die als extreme Entwicklung des Subjektivismus betrachtet wird (Schmitt 1991: 704) und "die das subjektive Ich mit seinem Bewusstseinsinhalt für das einzige Seiende hält." (Schmitt 1991: 673) Der methodische Solipsismus wie bei Descartes ist ein anderer Aspekt, von dem aus die außer dem Ich bestehende Wirklichkeit kritisiert wird. In "Der logische Aufbau der Welt" hält Rudolf Carnap nur die Gegenstände für real, die bewusst einem Subjekt zugehören (Vgl. Carnap 1999). 
(Precht1/Burkhard 1996: 481) verstanden. Hier knüpfen wir an das Dilemma des Muttersprachenverlustes an, unter dem die Protagonistin leidet, die sich nur an einige, muttersprachliche Wörter erinnern kann, die hauptsächlich mit ausgeprägten Erlebnissen verbunden sind wie die Wörter "Görmek", "Kaya gecirmek", "ISCI" (12) und "Ruh" (51). Diese Wörter leben noch in ihrem muttersprachlichen Gedächtnis und bilden eine neue Wirklichkeit, die die Muttersprache mosaikartig auf einzelne Wörter reduziert. Allerdings hat diese Wirklichkeit allein mit dem Subjekt der Protagonistin zu tun, mit anderen Worten zeigt die hier bestrebte Interpretation des Muttersprachenverlustes keine allgemeingültige Diagnose, denn "es gibt keine Ordnung der Dinge a priori" (Wittgenstein 1922: 5.634). Die koordinierte, solipsistische Wirklichkeit des Subjekts belebt einige Momente der muttersprachlichen Wirklichkeit durch das Erinnern erlebnisreicher Wörter. Diese erinnerten Wörter tauchen im Gedächtnis aus dem Unterbewusstsein auf. Von den Wörtern, an die sich die Ich-Erzählerin erinnert, sind zwei im Traum (12) aufgetaucht, doch im Traum kommen diese Wörter auch in Zusammenhang mit einem Erlebnis vor. Ein anderes Wort (11f) wird durch die direkte Wahrnehmung des türkischen Wortes in der Fremde erinnert.

\subsubsection{Anwendung, Virtualität und Aktualität}

Andere Wörter werden nicht erinnert, da sie nicht verwendet werden. "Wird ein Zeichen nicht gebraucht, so ist es bedeutungslos." (Wittgenstein 1922: 3.328, Hervorhebung im Original) Die Bedeutung eines Zeichens hängt von dessen Anwendung durch den ab, der es anwendet. Die Bedeutung eines Zeichens ist somit subjektiv beladen. Semiotisch hat das Zeichen zwei Grundeigenschaften, die Virtualität und Aktualität (Buch/ Stenschke 2008: 20). Virtuell ist das Zeichen in der Vorstellung, sobald es in der Realität verwendet wird, wird es aktuell. Das Zeichen ist bilateral, es hat Ausdruck und Inhalt. Der Inhalt der erinnerten Wörter bringt in der Vorstellung der Protagonistin deren Ausdruck hervor. Die nicht erinnerten Wörter haben in ihrer neuen, muttersprachlichen Wirklichkeit weder Inhalt noch Ausdruck, da der Reiz der Anwendung fehlt. Dazu kommt die historische Abschaffung der arabischen Schrift durch Atatürk. "Dieses Verbot ist so, wie wenn die Hälfte von meinem Kopf abgeschnitten ist. [...] Gottseidank ich gehöre noch zu einer 
Transkulturelle Aspekte der Sprache als Identitätsstifter in Emine Özdamars „,Mutterzunge“

Generation, die mit vielen arabischen Wörtern aufgewachsen ist." (31) Beim Wort hat man die semiotische Hälfte der türkischen Sprache mit diesem Verbot abgeschafft, die mit einem Schlag ihre Schrift verloren hat. Daher sucht die Protagonistin im Arabischen genetisch verfolgend den Weg zu ihrer Mutterzunge. Arabisch verkörpert die grafische Seite des Alttürkischen ohne Laut bzw. ohne praktische Anwendung. Auf Deutsch verständigen sich beide ausländischen Protagonisten, da es in der realen Gegenwart gebraucht wird, während ihre Muttersprachen der Vergangenheit angehören.

\subsection{Politische Einstellung und Sprachverlust}

In der Heimat beginnt der Sprachverlust bei der Protagonistin schon durch ihre oppositionelle Einstellung gegenüber der politischen Lage, der Unterdrückung der Opposition, in der Türkei, im Zug derer mehrere Aktivisten gebrandmarkt (10) oder getötet (11) werden. Ein Oppositioneller namentlich Mahir war "in den Zeitungen als Stadtbandit bekannt gemacht [...] In den Tagen hatten sie Mahir mit Kugeln getötet" (14). Eine Generation fühlt gewisse Bitterkeit der Heimat gegenüber. Die Erinnerungen an ständige Angst, das Leiden und die Klagen um die Opfer sind so bedrückend, dass sie den Entfremdungsprozess bis zum Verlust der Muttersprache beschleunigen.

Eine Antipathie gegen alles Einheimische bemächtigt sich dieser Jugendlichen. "Man hat ihnen die Milch, die sie aus ihren Müttern getrunken haben, aus ihrer Nase rausgeholt." (14) Diese negative Auffassung von der eigenen Heimat bewegt die Ich-Erzählerin dazu, bei einer Kontrolle zu behaupten: "Ich bin Italienerin." (12) Sie lehnt nicht nur ihre Herkunft, sondern auch ihre Sprache ab. "Ich bin unglücklich in meiner Sprache. [...] Die Wörter sind krank. [...] Wie lange braucht ein Wort, um wieder gesund zu werden? Man sagt, in fremden Ländern verliert man die Muttersprache. Kann man nicht auch in seinem eigenen Land die Muttersprache verlieren?" (Özdamar 2001: 128f) Ihre Welt findet sich mit der Muttersprache nicht ab. Özdamar weist damit auf die Möglichkeit hin, dass man seine Muttersprache auch in eigener Heimat verlieren kann. Die politische Unterdrückung ist neben der Abschaffung der arabischen Schrift ein anderer Grund für den Muttersprachenverlust. 
8. $\quad$ Fazit

Die Transkulturalität geht über die traditionellen, abgrenzenden Konzepte von Kulturen als kugelhafte, gegeneinander abzugrenzende Größen hinaus und betont hingegen die permeative Bewandtnis der Kulturen, die sich heutzutage immer mehr verflechten. Die Dichotomie "Nomade und Monade", worauf Welsch das Augenmerk lenkt, weist genau auf die Grenze zwischen dem Modell der Transkulturalität und dem Modell von Inter- und Multikulturalität hin (Vgl. Welsch 2010: 46). Genau von diesem Modell der Transkulturalität geht Özdamar aus, die die migrationsbedingte Durchdringung der deutschen Kultur nicht als Identitätsgefährdung begreift. Wenn von Identitätsstörung die Rede ist, dann soll man nach deren Ursachen schon in der eignen Heimat und nicht in der Fremde suchen, die eine neue Wirklichkeit vorschreibt und dementsprechend eine neue Identitätsentwicklung fordert.

Der Verlust der Muttersprache, an dem die eigene Heimat mitschuldig ist, soll in der Fremde nicht als Leid verstanden werden, sondern als Gewinn einer neuen "anatürlichen Sprache" (Ben-Chorin 1988: 47), die die eigene Identität nicht bedroht sondern bereichert und profiliert. Diese neue Sprache kann die Muttersprache nicht ersetzen, sie ist eher eine neue sprachliche Identität, die als eine eigenwillige Entscheidung $\mathrm{zu}$ betrachten ist. In der Fremdsprache stiftet sich die Protagonistin in "Mutterzunge" neue Identität durch eine abweichende Grammatikalität im Deutschen, mit der sogar Özdamar als Schriftstellerin zufrieden ist, durch die Relativierung heiliger, islamischer Texte, womit sie sich von einer kugelhaften, religiösen Identität entfernt und gewisse Flexibilität aufweist, und durch die Verflechtung multilingualer, transkultureller Momente, die sich auf inhaltlicher Ebene durch die Mischung der Sprachen im Text und auf formaler Ebene durch deren Zusammenkommen in einem Makrotext auf Deutsch vollziehen. Özdamar hybridisiert im Text drei Sprachen. Die Fremdsprache erfüllt der Protagonistin eine zufriedenstellende, sprachliche Identität, während ihre muttersprachliche Identität nur Kindheit ohne Gegenwart hat und sie ihre mit dem Arabischen als alter Schriftsprache verbundene Identität eine ausschließlich theologische Natur einräumt. 
Transkulturelle Aspekte der Sprache als Identitätsstifter in Emine Özdamars „,Mutterzunge“

Die Studie hat versucht, die transkulturellen Aspekte der Sprache in Textauslegungsmomenten aufgrund des kulturwissenschaftlichen Verfahrens "close reading" herauszufinden und die Ursachen für den Muttersprachverlust anhand des komplementären Ansatzes "wide reading" (Vgl. Nünnig 2010: 294ff) zu ermitteln beruhend auf Wittgensteins Sprachphilosophie.

Wegen dem Aufhören, auf der Muttersprache $\mathrm{zu}$ denken und muttersprachliche Wörter $\mathrm{zu}$ verwenden, der neu koordinierten, solipsistischen, deutschen Wirklichkeit und schließlich der politischen Unterdrückung bleibt die Muttersprache deaktiviert. Sie verschwindet nicht oder geht nicht zugrunde, sondern ihre Welt hört auf, bis sie wieder angeregt wird. "Wie auch beim Tod die Welt sich nicht ändert, sondern aufhört." (Wittgenstein 1922: 6.431) Weil die Muttersprache nicht von einer aktiven, mentalen Logik ausgehen kann, kann die Protagonistin nur in der Fremdsprache Sätze aussprechen und in der Muttersprache lediglich einzelne Wörter. Am Ende begnügt sich die Ich-Erzählerin mit der mosaikartigen, muttersprachlichen Wirklichkeit und beteuert, dass sie lediglich "Wörtersammlerin" (51) ist. Damit beendet sie ihre Suche nach der Muttersprache als vollständige Wirklichkeit.

\section{Literaturliste}

AHLZWEIG, Claus (1994): Muttersprache - Vaterland. Die deutsche Nation und ihre Sprache. Opladen: Westdeutscher Verlag.

AL-SLAIMAN, Mustafa (1997): Literatur in Deutschland am Beispiel arabischer Autoren, zur Übertragung und Vermittlung von Kulturrealien. Bezeichnungen in der Migranten-und Exilliteratur. In: BLEICHER, Thomas (Hg.): Literatur der Migration. Mainz: Donata Kinzelbach Verlag. S. 88-99.

BEN-CHORIN, Schalom (1981): Sprache als Heimat. In: Nasarski, Peter (Hg.): Sprache als Heimat. Auswanderer erzählen. Schriftenreihe der Internationalen Assoziation Deutschsprachiger Medien [IADM]. Berlin / Bonn: Westkreuz-Verlag. S. 12-23.

BLIOUMI, Aglaia (2002): Vorwort. In: dies. (Hg.): Migration und Interkulturalität in neueren literarischen Texten. München: Iudicium. S. 7-14.

BÖHM, Andrea (1999): Zwischen die Fronten geraten. Unter: www.zeit.de/1999/08/199908.mutterzunge .xml. Am 18. Feb. 1999.

BUCH, Albert / STENSCHKE, Oliver (2008): Germanistische Linguistik. 2.Aufl. Tübingen: Günter Narr. 


\section{Mahmoud Fouad}

CARNAP, Rudolf (1999): Der logische Aufbau der Welt.. Hamburg: Felix Meiner Verlag. (Erste Aufl. Wien: 1928)

GÖRLING, Reinhold. (1997): Heterotopia. Lektüren einer interkulturellen Literaturwissenschaft. München: Wilhelm Fink.

DUFRESNE, Marion (2006): Emine Sevgi Özdamar Mutter(s)zunge. Der Weg zum eigenen Ich. In: Germanica. Nr. 38. S. 115-128. Auch unter http://germanica.revues.org/373. S. 1-11 (Zitat im Text aus der digitalen Version)

ERIKSON, Erik H. (1992): Kindheit und Gesellschaft. 11. Aufl. Stuttgart: Klett-Cotta.

HOFMANN, Micheal (2006): Interkulturelle Literaturwissenschaft. Eine Einführung. Paderborn: Wilhelm Fink Verlag.

IVO, Hubert (1994): Muttersprache Identität Nation. Sprachliche Bildung im Spannungsfeld von einheimisch und fremd. Opladen: Westdeutscher Verlag.

JEPPESEN, Helle (2008): Sprache ist Identität. Unter: http://dw.de/p/DAHw. Am 24. Feb. 2008.

KEUPP, Heiner (1998): Diskursarena Identität: Lernprozesse in der Identitätsforschung. In: Ders. / HOFER, Renate (Hg.): Identitätsarbeit heute. Klassische und aktuelle Perspektiven der Identitätsforschung. 2. Aufl. Frankfurt/M.: Suhrkamp. S. 11-39.

KEUPP, Heiner (1999): Identitätskonstruktionen. Das Patchwork der Identitäten in der Spätmoderne. Reinbek bei Hamburg: Rowohlt.

MAHER, Moustafa (2007): Sinngemäße deutsche Übersetzung des Heiligen Koran. ArabischDeutsch. 2. Aufl. Kairo: Al-Ahram.

NASARSKI, Peter (Hg.) (1981): Sprache als Heimat. Auswanderer erzählen. Schriftenreihe der Internationalen Assoziation Deutschsprachiger Medien [IADM]. Berlin, Bonn: WestkreuzVerlag.

NÜNNING, Vera/ NÜNNING, Ansgar (Hg.) (2010): Methoden der literatur- und kulturwissenschaftlichen Textanalyse. Stuttgart/Weimar: Metzler.

ÖZDAMAR, Emine (1993): Schwarzauge und sein Esel. Unter: http://www.zeit.de/1993/09/schwarzauge-und-sein-esel. Am 26. Feb. 1993.

ÖZDAMAR, Emine (2001): Meine deutschen Worte haben keine Kindheit. Eine Dankrede. In: Dies.: Der Hof im Spiegel. Köln: Kiepenheuer\&Witsch. S. 125-132.

ÖZDAMAR, Emine Sevgi (1992): Das Leben ist eine Karawanserei hat zwei Türen aus einer kam ich rein aus der anderen ging ich raus. Köln: Kiepenheuer \& Witsch.

ÖZDAMAR, Emine (2010): Mutterzunge. 4. Aufl. Berlin: Rotbuch.

ÖZDAMAR, Emine (1994): Gespräch mit Deniz Göktürk. In: GÖKTÜRK, Deniz: Muttikültürelle Zungenbrecher, Literatütken aus Deutschland Nischen. In: Sirene. Nr. 12/13. S. 77-92.

PRECHTL, Peter / Burkhard, Franz-Peter (Hg.) (1996): Metzler Philosophie Lexikon, Begriffe und Definitionen. Stuttgart / Weimar: Metzler.

RIEGER，Stefan/SCHAHADAT, Schamma/WEINBERG, Manfred (1999): Interkulturalität zwischen Inszenierung und Archiv. Tübingen: Günter Narr. 
Transkulturelle Aspekte der Sprache als Identitätsstifter in Emine Özdamars „,Mutterzunge“

SCHISKOFF, Georgi (1952/53): Heimatlose in der Sprache. In: Wirkendes Wort. Deutsches Sprachschaffen in Lehre und Leben. 3. Jahrgang. 1952/53. S. 65-67.

SCHMIDT, Heinrich (1991): Philosophisches Wörterbuch. 22. Aufl. Stuttgart: Alfred Körner.

STEINMÜLLER, Ulrich (1984): Muttersprache. In: Georg Auernheimer (Hg.): Handwörterbuch Ausländerarbeit. Weinheim, Basel: Beltz. S. 241-244.

WEISGERBER, Leo (1938): Ist Muttersprache eine Germanische oder eine Romanische Prägung? In: Beiträge zur Geschichte der deutschen Sprache und Literatur. Bd. 62. S. 428-437.

WEISGERBER, Leo (1957): Die Muttersprache im Aufbau unserer Kultur. Bd. III der gesammelten Schriften "Von den Kräften der deutschen Sprache". Düsseldorf: Pädagogischer Verlag Schwann.

WELSCH, Wolfgang (2010): Hochschule als transkultureller Raum? Kultur, Bildung und Differenz in der Universität. Bielefeld: transcript-Verlag.

WERMKE, Matthias (Hg.) (2006): Duden Deutsches Universalwörterbuch. 6. Aufl. Mannheim: Duden Verlag. [CD-ROM]

WITTGENSTEIN, Ludwig (1922): Tractatus logica-philosophicus. Logisch-philosophische Abhandlung. In: Ders.: Schriften. Band 1. Frankfurt a/M.: Suhrkamp.

ZIMMERMANN, Harro (1986): Türkische Selbstpersiflage. In: Frankfurter Rundschau. Am 6. Mai 1986. S.7.

Arabische Literatur

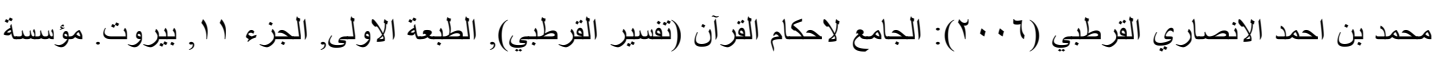

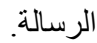

Al-KORTOBY, Mohamed (2006): Al-Kortoby Koranauslegung. 1. Aufl. Bd. 11. Beirut: AlResalah. (Sure "Yousef", Vers 23). 